\title{
Detection of extended very-high-energy $\gamma$-ray emission towards the young stellar cluster Westerlund 2
}

F. Aharonian ${ }^{1}$, A. G. Akhperjanian² ${ }^{2}$ A. R. Bazer-Bachi ${ }^{3}$, M. Beilicke ${ }^{4}$, W. Benbow ${ }^{1}$, D. Berge $^{1, \star}$, K. Bernlöhr ${ }^{1,5}$, C. Boisson ${ }^{6}$, O. Bolz ${ }^{1}$, V. Borrel ${ }^{3}$, I. Braun ${ }^{1}$, E. Brion ${ }^{7}$, A. M. Brown ${ }^{8}$, R. Bühler ${ }^{1}$, I. Büsching ${ }^{9}$, T. Boutelier ${ }^{17}$, S. Carrigan ${ }^{1}$, P. M. Chadwick ${ }^{8}$, L.-M. Chounet ${ }^{10}$, G. Coignet $^{11}$, R. Cornils ${ }^{4}$, L. Costamante ${ }^{1,23}$, B. Degrange ${ }^{10}$, H. J. Dickinson ${ }^{8}$, A. Djannati-Ataï ${ }^{12}$, L. O'C. Drury ${ }^{13}$, G. Dubus ${ }^{10}$, K. Egberts ${ }^{1}$, D. Emmanoulopoulos ${ }^{14}$, P. Espigat ${ }^{12}$, C. Farnier ${ }^{15}$, F. Feinstein ${ }^{15}$, E. Ferrero ${ }^{14}$, A. Fiasson ${ }^{15}$, G. Fontaine ${ }^{10}$, Seb. Funk ${ }^{5}$, S. Funk ${ }^{1}$, M. Füßling ${ }^{5}$, Y. A. Gallant ${ }^{15}$, B. Giebels ${ }^{10}$, J. F. Glicenstein ${ }^{7}$, B. Glück ${ }^{16}$, P. Goret ${ }^{7}$, C. Hadjichristidis ${ }^{8}$, D. Hauser ${ }^{1}$, M. Hauser ${ }^{14}$ G. Heinzelmann ${ }^{4}$, G. Henri ${ }^{17}$, G. Hermann ${ }^{1}$, J. A. Hinton ${ }^{1,14, \star \star}$, A. Hoffmann ${ }^{18}$, W. Hofmann ${ }^{1}$, M. Holleran ${ }^{9}$, S. Hoppe ${ }^{1}$, D. Horns ${ }^{18}$, A. Jacholkowska ${ }^{15}$, O. C. de Jager ${ }^{9}$, E. Kendziorra ${ }^{18}$, M. Kerschhaggl ${ }^{5}$, B. Khélifi ${ }^{10,1}$,' Nu. Komin ${ }^{15}$, K. Kosack ${ }^{1}$, G. Lamanna ${ }^{11}$, I. J. Latham ${ }^{8}$, R. Le Gallou ${ }^{8}$, A. Lemière ${ }^{12}$, M. Lemoine-Goumard ${ }^{10}$, T. Lohse ${ }^{5}$, J. M. Martin ${ }^{6}$, O. Martineau-Huynh ${ }^{19}$, A. Marcowith ${ }^{3,15}$, C. Masterson ${ }^{1,23}$, G. Maurin ${ }^{12}$, T. J. L. McComb ${ }^{8}$, E. Moulin ${ }^{15,7}$, M. de Naurois ${ }^{19}$, D. Nedbal ${ }^{20}$, S. J. Nolan ${ }^{8}$, A. Noutsos ${ }^{8}$, J-P. Olive ${ }^{3}$, K. J. Orford ${ }^{8}$, J. L. Osborne ${ }^{8}$, M. Panter ${ }^{1}$, G. Pelletier ${ }^{17}$, P.-O. Petrucci ${ }^{17}$, S. Pita ${ }^{12}$, G. Pühlhofer ${ }^{14}$, M. Punch ${ }^{12}$, S. Ranchon ${ }^{11}$, B. C. Raubenheimer ${ }^{9}$, M. Raue ${ }^{4}$, S. M. Rayner ${ }^{8}$, A. Reimer ${ }^{\star \star \star}$, O. Reimer $^{\star \star \star}$, J. Ripken ${ }^{4}$, L. Rob ${ }^{20}$, L. Rolland ${ }^{7}$, S. Rosier-Lees ${ }^{11}$, G. Rowell ${ }^{1, \dagger}$, V. Sahakian ${ }^{2}$, A. Santangelo ${ }^{18}$, L. Saugé ${ }^{17}$, S. Schlenker ${ }^{5}$, R. Schlickeiser ${ }^{21}$, R. Schröder ${ }^{21}$, U. Schwanke ${ }^{5}$, S. Schwarzburg ${ }^{18}$, S. Schwemmer ${ }^{14}$, A. Shalchi ${ }^{21}$, H. Sol ${ }^{6}$, D. Spangler ${ }^{8}$, F. Spanier ${ }^{21}$, R. Steenkamp ${ }^{22}$, C. Stegmann ${ }^{16}$, G. Superina ${ }^{10}$, P. H. Tam ${ }^{14}$, J.-P. Tavernet ${ }^{19}$, R. Terrier ${ }^{12}$, M. Tluczykont ${ }^{10,23},{ }^{\ddagger}$, C. van Eldik $^{1}$, G. Vasileiadis ${ }^{15}$, C. Venter ${ }^{9}$, J. P. Vialle ${ }^{11}$, P. Vincent ${ }^{19}$, H. J. Völk ${ }^{1}$, S. J. Wagner ${ }^{14}$, and M. Ward ${ }^{8}$

\section{(Affiliations can be found after the references)}

Received 15 December 2006 / Accepted 5 March 2007

\section{ABSTRACT}

Aims. Results from $\gamma$-ray observations by the HESS telescope array in the direction of the young stellar cluster Westerlund 2 are presented. Methods. Stereoscopic imaging of Cherenkov light emission of $\gamma$-ray induced showers in the atmosphere is used to study the celestial region around the massive Wolf-Rayet (WR) binary WR 20a. Spectral and positional analysis is performed using standard event reconstruction techniques and parameter cuts.

Results. The detection of a new $\gamma$-ray source is reported from HESS observations in 2006. HESS J1023-575 is found to be coincident with the young stellar cluster Westerlund 2 in the well-known HII complex RCW 49. The source is detected with a statistical significance of more than $9 \sigma$, and shows extension beyond a point-like object within the HESS point-spread function. The differential $\gamma$-ray spectrum of the emission region is measured over approximately two orders of magnitude in flux.

Conclusions. The spatial coincidence between HESS J1023-575 and the young open cluster Westerlund 2, hosting e.g. the massive WR binary WR 20a, requires one to look into a variety of potential models to account for the observed very-high-energy (VHE) $\gamma$-ray emission. Considered emission scenarios include emission from the colliding wind zone of WR 20a, collective stellar winds from the extraordinary ensemble of hot and massive stars in the stellar cluster Westerlund 2, diffusive shock acceleration in the wind-blown bubble itself, and supersonic winds breaking out into the interstellar medium (ISM). The observed source extension argues against a single star origin of the observed VHE emission.

Key words. ISM: individual objects: HESS J1023-575 - ISM: individual objects: RCW 49 (NGC 3247, G284.3-0.3) - stars: Wolf-Rayet stars: individual: WR 20a - gamma rays: observations - ISM: HII regions

\section{Introduction}

The prominent giant HII region RCW 49 (also NGC 3247), and its ionizing cluster Westerlund 2 are located towards the

* Now at CERN, Geneva, Switzerland.

$\star \star$ Now at School of Physics \& Astronomy, University of Leeds, Leeds LS2 9JT, UK.

$\star \star \star$ Now at Stanford University, HEPL \& KIPAC, Stanford, CA 943054085, USA, e-mail: olr@stanford.edu

Now at School of Chemistry \& Physics, University of Adelaide, Adelaide 5005, Australia.

Now at DESY Zeuthen. outer edge of the Carina arm of our Milky Way. RCW 49 is a luminous, massive star formation region, and has been extensively studied at various wavelengths. After the initial report based on photographic images by Rodgers et al. (1960), the region was observed at X-ray energies with Einstein (Hertz \& Grindlay 1984; Goldwurm et al. 1987), ROSAT (Belloni \& Mereghetti 1994), and recently Chandra, which discovered $\sim 500$ point sources in the vicinity of RCW 49 (Tsujimoto et al. 2004), with $\sim 100$ of them spatially coincident with the central open stellar cluster Westerlund 2 (Townsley et al. 2005). Mid-infrared measurements with Spitzer revealed still ongoing massive star formation in RCW 49 (Whitney et al. 2004). The 
regions surrounding Westerlund 2 appear evacuated by stellar winds and radiation. The surrounding dust is distributed in fine filaments, knots, pillars, bubbles, and bow shocks throughout the rest of the HII complex (Churchwell et al. 2004; Conti \& Crowther 2004). Radio continuum observations by ATCA at 1.38 and $2.38 \mathrm{GHz}$ indicate two wind-blown shells in the core of RCW 49 (Whiteoak \& Uchida 1997): one surrounding the central region of Westerlund 2, the other the prominent WR star WR 20b. The distance to RCW 49 is still uncertain and values range between $2.2 \mathrm{kpc}$ (Brand \& Blitz 1993) up to $7.9 \mathrm{kpc}$ (Moffat et al. 1991), whereas intermediate values of $4.2 \mathrm{kpc}$ from $21 \mathrm{~cm}$ absorption line profile measurements (McClure-Griffiths et al. 2001), $5.75 \mathrm{kpc}$ from the distance estimate towards the prominent WR star WR 20a (Van der Hucht 2001), and $6.4 \mathrm{kpc}$ from photometric measurements (Carraro \& Munari 2004) correspond to more recent refinements. Finally, Rauw et al. (2007) presented a compelling re-determination of the distance to Westerlund 2 by spectro-photometric measurements of 12 cluster member O-type stars of $(8.3 \pm 1.6) \mathrm{kpc}$. This value is in very good agreement with the $(8.0 \pm 1.0) \mathrm{kpc}$ as measured by Rauw et al. (2005), determined from the light curve of the eclipsing binary WR 20a. In summary, we adopt the distance value of the weighted mean of $(8.0 \pm 1.4) \mathrm{kpc}$ (Rauw et al. 2007) throughout the manuscript, thereby associating WR 20a as a cluster member of Westerlund 2.

Westerlund 2 (Westerlund 1960) contains an extraordinary ensemble of hot and massive stars, presumably at least a dozen early-type O-stars, and two remarkable WR stars. Two WolfRayet (WR) stars have been found within RCW 49, WR 20b at $\alpha_{2000}=10^{\mathrm{h}} 24^{\mathrm{m}} 18.4, \delta_{2000}=-57^{\circ} 48^{\prime} 30^{\prime \prime}$, and WR 20a at $\alpha_{2000}=10^{\mathrm{h}} 23^{\mathrm{m}} 58^{\mathrm{s}} .0, \delta_{2000}=-57^{\circ} 45^{\prime} 49^{\prime \prime}$ (Van der Hucht 2001), both first reported as WR stars by Shara et al. (1991), the latter believed to be a member of the stellar cluster Westerlund 2 . Only recently was WR20a established to be a binary: both Rauw et al. (2004) and Bonanos et al. (2004) presented solutions for a circular orbit with a period of 3.675 , and 3.686 days, respectively. Based on the the orbital period, the minimum masses have been found to be $(83 \pm 5) M_{\odot}$ and $(82 \pm 5) M_{\odot}$ for the primary and secondary components, respectively (Rauw et al. 2005). This classifies the WR binary WR 20a as the most massive of all confidently measured binary systems in our Galaxy. An orbital inclination of $\sim 75^{\circ}$ was inferred from photospheric eclipses (Bonanos et al. 2004), and the spectral classification was subsequently refined as a binary consisting of two WN6ha stars (Rauw et al. 2005). The supersonic stellar winds of both WR stars collide, and a wind-wind interaction zone forms at the stagnation point with a reverse and forward shock. In a detached binary system like WR 20a, the colliding wind zone lies between the two stars, and is heavily skewed by Coriolis forces. The wind velocity at the stagnation point is likely lower than in other positions due to the radiative inhibition mechanism (Stephens \& Pollock 1994) caused by the companion's stellar photon field. In a close binary system the winds can only be radiatively accelerated to a fraction of their expected $v_{\infty} \sim 2800 \mathrm{~km} \mathrm{~s}^{-1}$, and a comparatively low pre-shock wind velocity of $\sim 500 \mathrm{~km} \mathrm{~s}^{-1}$ follows (Rauw et al. 2005).

WR stars are commonly thought to represent an evolved phase for the most massive stars in our Galaxy, however the most luminous hydrogen-rich WR stars may still be thought of as the most massive, main-sequence stars (Moffat et al. 2006), exhibiting strong, broad WR-like emission lines at optical wavelengths. Some WR stars are already established as non-thermal radio emitters, whereas claimed associations with $\gamma$-ray emission remain to be confirmed. It has been suggested that non-thermal radio emission observed in a significant fraction of the massive stars could be related to colliding winds in binaries (Dougherty \& Williams 2000). An important question is therefore whether all massive stars which produce non-thermal radio emission are indeed binaries. Another relevant issue is whether the relativistic electrons which produce the observed non-thermal (synchrotron) radio emission also produce detectable (inverse Compton) highenergy emission. Synchrotron emission has not been detected from the WR 20a system, presumably because of free-freeabsorption in the optically thick stellar winds along the line of sight. However, WR 20a has been detected in X-rays (Mereghetti $\&$ Belloni 1996). With a flux of the order of $10^{-12} \mathrm{erg} \mathrm{cm}^{-2} \mathrm{~s}^{-1}$, the soft X-ray emission is consistent with the predicted intensity for the thermal radiation from the shock-heated material in the stellar wind (Pittard \& Stevens 2002). However, non-thermal and thermal components of the X-ray emission are currently indistinguishable. In high-energy $\gamma$-rays, three unidentified EGRET sources have been found in the wider vicinity of RCW 49, 3EG J1013-5915, 3EG J1014-5705, and 3EG J1027-5817 (Hartman et al. 1999). These EGRET sources are characterized by both source confusion, and indication of extended emission, therefore viable counterparts are not established readily.

It has been suggested that in massive binary systems highenergy $\gamma$-rays are produced either by optically-thin inverse Compton scattering of relativistic electrons with the dense photospheric stellar radiation fields in the wind-wind collision zone (Eichler \& Usov 1993; White \& Chen 1995; Benaglia \& Romero 2003; Reimer et al. 2006; Pittard \& Dougherty 2006), or in hadronic emission scenarios. Here, $\gamma$-rays are either directly produced in neutral pion decays, with the mesons produced by inelastic interactions of relativistic nucleons with the wind material (White \& Chen 1992; Benaglia \& Romero 2003; Torres et al. 2004; Reimer et al. 2006; Pittard \& Dougherty 2006), or alternatively are produced in cascade models (Bednarek 2005). In the latter scenario, $\gamma$-radiation originates from inverse-Compton pair cascades, which are initiated by high-energy neutral pion decay photons (from nucleon-nucleon interactions in the stellar winds). Due to the dense radiation field at the wind collision zone in the close binary WR 20a, $\gamma$-rays of a few tens of $\mathrm{GeV}$ are optically thick to photon-photon absorption, and pair cascades are unavoidable. If those nuclei reach sufficiently high energies (despite the expected weak shock in the extremely dense radiative environment), the projectile nucleons themselves are produced by photodisintegration of heavy nuclei in the radiation fields of the massive WR-stars (Bednarek 2005). For a high magnetic field of order $10^{3} \mathrm{G}$, particles could be energized up to $\sim 10^{15} \mathrm{eV}$ (adopting typical WR-wind parameters) in the magnetic field reconnection scenario, and up to $10^{3} \mathrm{GeV}$ if first-order Fermi acceleration takes place there. The maximum power extractable from WR 20a must be a fraction of the kinetic wind energy provided by the system, estimated to be $\sim 10^{37} \mathrm{erg} / \mathrm{s}$.

Detectable VHE $\gamma$-radiation from the WR 20a binary system is so far only predicted in the pair cascade model, although detailed modeling of the WR 20a system in the other scenarios is still pending. For VHE $\gamma$-rays, photon-photon absorption will diminish the observable flux from a close binary system such as WR 20a (Bednarek 2005; Dubus 2006).

Here we report on the first VHE $\gamma$-ray observations towards the WR binary WR 20a, and its immediate surroundings with the HESS telescopes. The observations and analysis yield the detection of a new source, HESS J1023-575. This source is found to coincide with the central region of RCW 49, and accordingly, with the stellar cluster Westerlund 2, which hosts the WR binary 
WR 20a. The results and possible interpretation of these findings are presented in the following.

\section{HESS oservations and data analysis}

The HESS (High Energy Stereoscopic System) collaboration operates a telescope system of four imaging atmospheric Cherenkov telescopes located in Namibia $\left(23^{\circ} 16^{\prime} 17^{\prime \prime} \mathrm{S} 16^{\circ} 29^{\prime} 58^{\prime \prime} \mathrm{E}\right)$, at $1800 \mathrm{~m}$ above sea level. Each telescope has a tesselated spherical mirror with $13 \mathrm{~m}$ diameter and $107 \mathrm{~m}^{2}$ area, and a telescope spacing of $120 \mathrm{~m}$. Cherenkov light emitted by extended air showers in the atmosphere is imaged by a high resolution camera (960 photo-multipliers, pixel size $0.16^{\circ}$ ). Shower parameters and the primary particle type are determined by image reconstruction (Aharonian et al. 2004, 2006b). The telescopes are operated in coincidence mode, which requires a trigger from at least two out of the four telescopes (Funk et al. 2004). The HESS telescope array achieves a point source sensitivity above $1 \mathrm{TeV}$ of $<2.0 \times 10^{-13} \mathrm{~cm}^{-2} \mathrm{~s}^{-1}$ ( $1 \%$ of the flux from the Crab nebula) for a $5 \sigma$ detection in a $25 \mathrm{~h}$ observation.

The dataset described here consists of $14 \mathrm{~h}$ ( $12.9 \mathrm{~h}$ live time) of data taken between March and July 2006, either on the nominal source location of WR 20a or overlapping data from the ongoing Galactic plane survey. Quality selections were imposed on the data, excluding those taken during bad weather or with hardware irregularities. The data have been obtained in wobblemode observations, where the telescopes are pointed offset from the nominal source location to allow for simultaneous background estimation. The wobble offsets for these observations range from $0.5^{\circ}$ to $2^{\circ}$, with the majority of data taken with wobble offset less than $0.8^{\circ}$. The zenith angles range between $36^{\circ}$ and $53^{\circ}$, resulting in an energy threshold of $380 \mathrm{GeV}$ for the analysis.

The data have been analyzed using the HESS standard Hillas analysis with standard cuts (image amplitude $>80$ p.e.). Optical efficiency corrections have been applied as described in Aharonian et al. (2006b). A point source analysis on the nominal position of WR 20a resulted in a clear signal with a significance of 6.8 standard deviations. Further investigations of the skymap of this region revealed an extended excess with a peak significance exceeding 9 standard deviations (Fig. 1). The position of the center of the excess was derived by fitting the two-dimensional point spread function (PSF) of the instrument folded with a Gaussian to the uncorrelated excess map: $\alpha_{2000}=10^{\mathrm{h}} 23^{\mathrm{m}} 18^{\mathrm{s}} \pm 12^{\mathrm{s}}, \delta_{2000}=-57^{\circ} 45^{\prime} 50^{\prime \prime} \pm 1^{\prime} 30^{\prime \prime}$. The systematic error in the source location is $20^{\prime \prime}$ in both coordinates. The number of excess events versus the squared angular distance to this position in comparison to the expectation for a point source (dashed line) is shown in Fig. 2. The excess is clearly extended beyond the nominal extension of the PSF. A fit of a Gaussian folded with the PSF of the HESS instruments gives an extension of $\sigma=0.18^{\circ} \pm 0.02^{\circ}$. The differential energy spectrum for photons inside the corresponding $85 \%$ containment radius of $0.39^{\circ}$ is shown in Fig. 3. It can be described by a power law $\left(\mathrm{d} N / \mathrm{d} E=\Phi_{0} \cdot(E / 1 \mathrm{TeV})^{-\Gamma}\right)$ with a photon index of $\Gamma=2.53 \pm 0.16_{\text {stat }} \pm 0.1_{\text {syst }}$ and a normalization at $1 \mathrm{TeV}$ of $\Phi_{0}=\left(4.50 \pm 0.56_{\text {stat }} \pm 0.90_{\text {syst }}\right) \times 10^{-12} \mathrm{TeV}^{-1} \mathrm{~cm}^{-2} \mathrm{~s}^{-1}$. The integral flux for the whole excess above the energy threshold of $380 \mathrm{GeV}$ is $(1.3 \pm 0.3) \times 10^{-11} \mathrm{~cm}^{-2} \mathrm{~s}^{-1}$. No significant flux variability could be detected in the data set. The fit of a constant function to the lightcurve binned in data segments of $28 \mathrm{~min}$ has a chance probability of 0.14 . The results were checked with independent analyses and were found to be in good agreement.

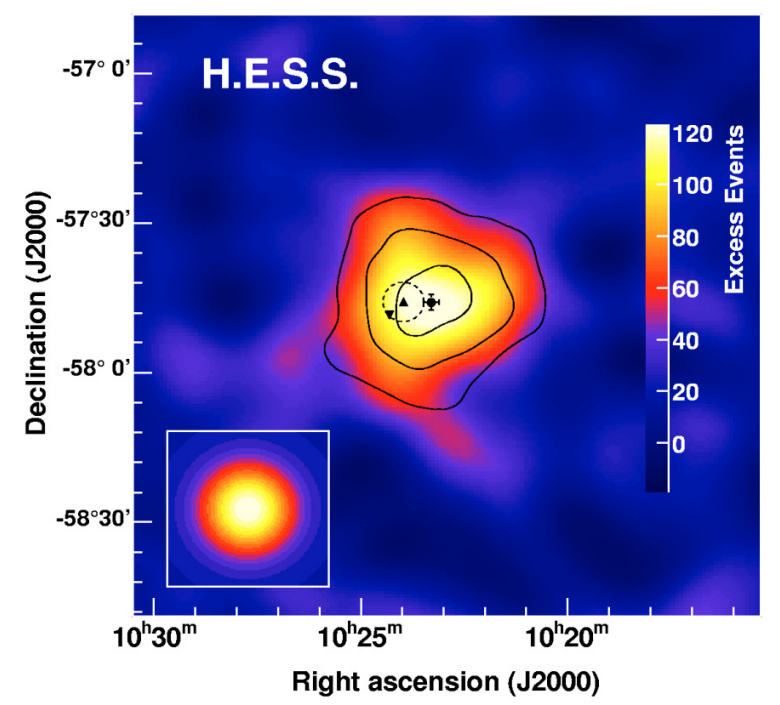

Fig. 1. Correlated excess sky map with an oversampling radius of $0.12^{\circ}$ of the Westerlund 2/RCW 49 region. The background in each bin is estimated using a ring around the test position. The map has been smoothed with a two-dimensional Gaussian of radius $0.04^{\circ}$ to reduce the effect of statistical fluctuations. Overlaid contours correspond to statistical significances of 5, 7, and $9 \sigma$, respectively, determined using the nominal oversampling radius for extended sources of $0.2^{\circ}$. The inlay in the lower left corner shows the excess distribution for a point-source derived from Monte Carlo data with the same zenith-angle and offset distribution as the data. The cross denotes the best fit position of the gamma-ray data with $1 \sigma$ statistical uncertainties. The WR binary WR 20a is indicated by an upright filled triangle in the Westerlund 2 stellar cluster (dashed circle), the reversed filled triangle denotes the location of WR $20 \mathrm{~b}$.

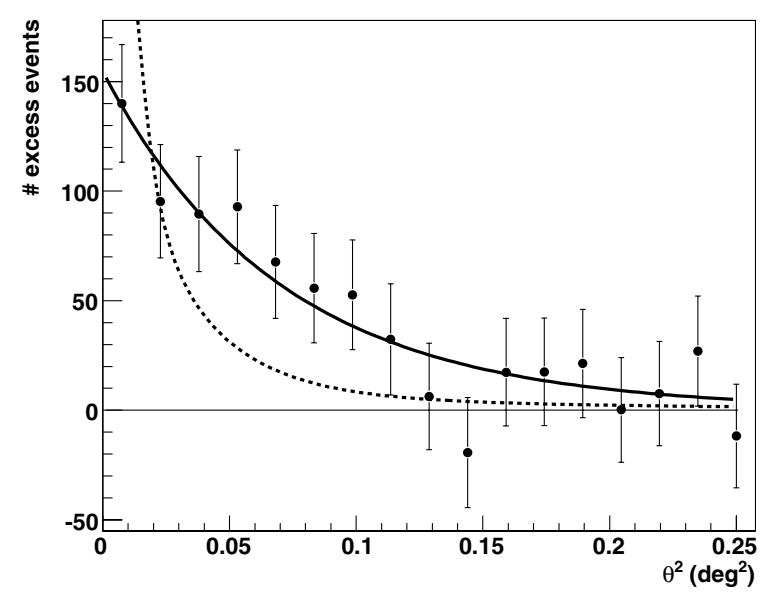

Fig. 2. Number of excess events versus the squared angular distance from the best fit position of the excess $\left(\theta^{2}\right)$. The dashed line shows the expectation for a point source derived from Monte Carlo data. The solid line is a fit of the PSF folded with a Gaussian $\left(\sigma=0.18^{\circ} \pm 0.02^{\circ}\right)$.

\section{HESS J1023-575 in the context of $\gamma$-ray emission scenarios}

The detection of VHE photons from the region RCW 49/Westerlund 2, characterized by a moderately hard power-law spectrum with an index $\Gamma \sim 2.5$ is indicative of the existence of extreme high-energy particle acceleration processes in this young ( 2-3 Myr; Piatti et al. 1998) star forming region. The estimated luminosity above $380 \mathrm{GeV}$ is $\sim 1.5 \times 10^{35} \mathrm{erg} / \mathrm{s}$ at a distance of $8 \mathrm{kpc}$, which corresponds to $0.5 \%$ of the total kinetic energy available from the colliding winds of WR 20a, 


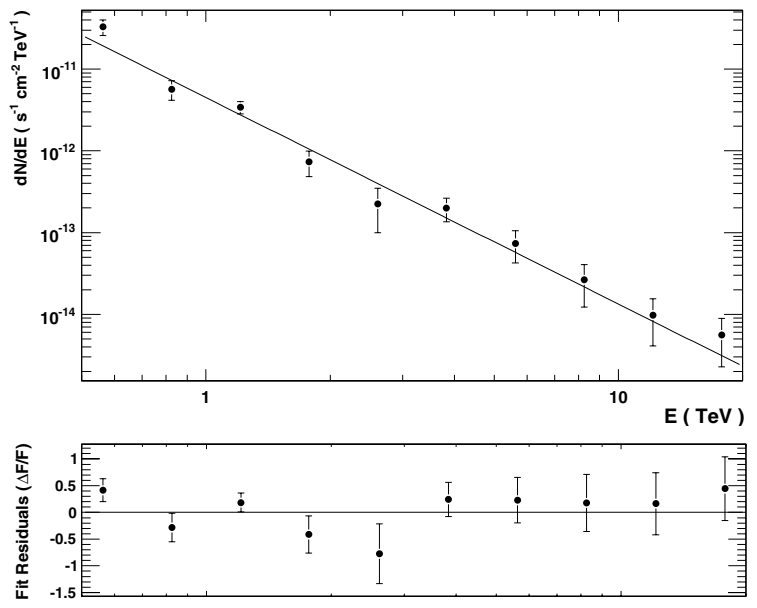

Fig. 3. Differential energy spectrum and fit residuals to a single powerlaw fit of HESS J1023-575 from photons inside the 85\% containment radius $\left(0.39^{\circ}\right)$ around the best fit position. The background is estimated with background regions of the same size and distance from the camera center as the signal region. The spectrum can be described by a power law with a photon index of $\Gamma=2.53 \pm 0.16_{\text {stat }} \pm 0.1_{\text {syst }}$ and a normalization at $1 \mathrm{TeV} \Phi_{0}=\left(4.50 \pm 0.56_{\text {stat }} \pm 0.90_{\text {syst }}\right) \times 10^{-12} \mathrm{TeV}^{-1} \mathrm{~cm}^{-2} \mathrm{~s}^{-1}$. The integral flux for the whole excess is $F(E>380 \mathrm{GeV})=(1.3 \pm 0.3) \times$ $10^{-11} \mathrm{~cm}^{-2} \mathrm{~s}^{-1}$

and $\sim 1.6 \%$ of the kinetic energy of WR 20b. With a projected angular size of submilliarcsecond scale, the WR 20a binary system, including its colliding wind zone, would appear as a point source for observations with the HESS telescope array. Unless there are extreme differences in the spatial extent of the particle distributions producing radio, X-ray, and VHE $\gamma$-ray emission, scenarios based on the colliding stellar winds in the WR 20 a binary system face the severe problem of accounting for a source extension of $0.18^{\circ}$ in the VHE waveband. At a nominal distance of WR 20a (8.0 kpc; Rauw et al. 2007), this source extension is equivalent to a diameter of $28 \mathrm{pc}$ for the emission region, consistent in size with theoretical predictions of bubbles blown from massive stars into the ISM (Castor et al. 1975). The spatial extension found for HESS J1023-575 contradicts emission scenarios where the bulk of the $\gamma$-rays is produced close to the massive stars. If VHE $\gamma$-rays were indeed produced near the massive stars in the binary, a regular modulation of the $\gamma$-ray flux due to absorption would be expected (Dubus 2006). Future observations of HESS J1023-575 will allow one to probe for such a pattern, which could discard or unambiguously label the origin of the VHE emission.

Alternatively, the emission could arise from collective effects of stellar winds in the Westerlund 2 cluster. Diffusive shock acceleration in cases where energetic particles experience multiple shocks (Klepach et al. 2000) can be considered for Westerlund 2. The stellar winds may provide a sufficiently dense target for high-energy particles (accelerated to hundreds of $\mathrm{TeV}$ in the winds themselves or driven by supernova explosions), allowing the production of $\pi^{0}$-decay $\gamma$-rays via inelastic pp-interactions. According to Rauw et al. (2007) Westerlund 2 may host about $4500 M_{\odot}$ in the form of stars with $M>M_{\odot}$. The mechanical energy injected through the stellar winds determines mainly the total energy available for collective wind effects, estimated to be about $5.4 \times 10^{37} \mathrm{erg} / \mathrm{s}$ at most. A collective wind scenario proposed by Domingo-Santamaria \& Torres (2006) predicts that the extension of a $\gamma$-ray source corresponds to the volume filled by the hot, shocked stellar winds. Variability is generally not expected since flux modulation of the winds of individual stars or binaries is believed to average out in a collective wind. Interestingly, a putative VHE source may not possess a non-thermal counterpart at $\mathrm{MeV} / \mathrm{GeV}$ energies in such a scenario, since convection will prevent low-energy particles from entering the wind. In both colliding wind zone and collective wind models, one would expect sub-TeV spectra that resemble those of Supernova remnants.

Magneto-hydrodynamic (MHD) particle acceleration (e.g. by multiple shocks or turbulence produced by supersonic flows) in a magnetized plasma may also be considered when particles penetrate into a dense medium. In such a case, the massive stellar winds of Westerlund 2 could accomplish three tasks: they could ensure sufficient particle injection into the turbulent plasma, feed the magnetic turbulence with energy via wind-wind interactions in the massive star association, or provide copious photons and dense material to serve as target for TeV-photon producing particle-photon and photon-photon interactions. Supershells, molecular clouds, and inhomogeneities embedded in the dense hot medium may serve as the targets for $\gamma$-ray production in Cosmic Ray interactions. Such environments have been already studied in the nonlinear theory of particle acceleration by largescale MHD turbulence (Bykov \& Toptygin 1987). Shocks and MHD turbulent motion inside a stellar bubble or superbubble can efficiently transfer energy to cosmic rays if the particle acceleration time inside the hot bubble is much shorter than the bubble's expansion time. X-ray observations of RCW 49 revealed, apart from point-like emission from individual stars, a diffuse component centered on the ionizing cluster, widely extending outside the core, with a soft component and a hard tail (Belloni \& Mereghetti 1994; Townsley et al. 2005). This indicates the existence of a wind-blown shell from the stars at the cluster core, filled with a hot $(\sim 0.1-3 \mathrm{keV})$ tenuous plasma shocked by turbulence. Two wind-blown bubbles are revealed from continuum radio observations: one around WR $20 \mathrm{~b}$ with a diameter of $\sim 4.1^{\prime}$ and an expansion velocity of $67 \mathrm{~km} \mathrm{~s}^{-1}$ (Shara et al. 1991), the other around the Westerlund 2 cluster center with a diameter of $\sim 7.3^{\prime}$ (Whiteoak \& Uchida 1997). A ridge of enhanced $\mathrm{GHz}$ radio emission lies between these two shells, and it has been suggested that this may represent a site of collision between these two shells (Whiteoak \& Uchida 1997). Most interestingly, the extent of a blister (Whiteoak \& Uchida 1997) on the western side of the Westerlund 2 bubble appears to be compatible in direction and location with the center of gravity of HESS J1023-575. Such a blister is indicative for rapid expansion into a low-density medium outside the wind-blown bubble. Figures 4 and 5 show overlays of the detection significance contours of HESS J1023-575 $(E>380 \mathrm{GeV})$ on the $B$-band image of the region as obtained from the Second Palomar Observatory Sky Survey (POSS-2), and on the $843 \mathrm{MHz}$ radio continuum image from the Molonglo Observatory Synthesis Telescope (MOST), respectively. Whereas the open cluster Westerlund 2 does not perfectly coincide with the peak of the emission seen at very high energy $\gamma$-rays (Fig. 4), the direction of the outbreak and spatial location of the blister is a better match (Fig. 5). The later association also relaxes constraints arising of the detection of HESS J1023-575 as an extended VHE $\gamma$-ray emitter.

Shock acceleration at the boundaries of the blister may enable particles to diffusively re-enter into the dense medium, thereby interacting in hadronic collisions and producing $\gamma$-rays. Consequently, a scenario as outlined in Völk (1983) for a supernova-driven expansion of particles into a low density medium may be applicable to the expanding stellar winds into the ambient medium. If one accepts such a scenario here, it might give the first observational support of $\gamma$-ray emission due 


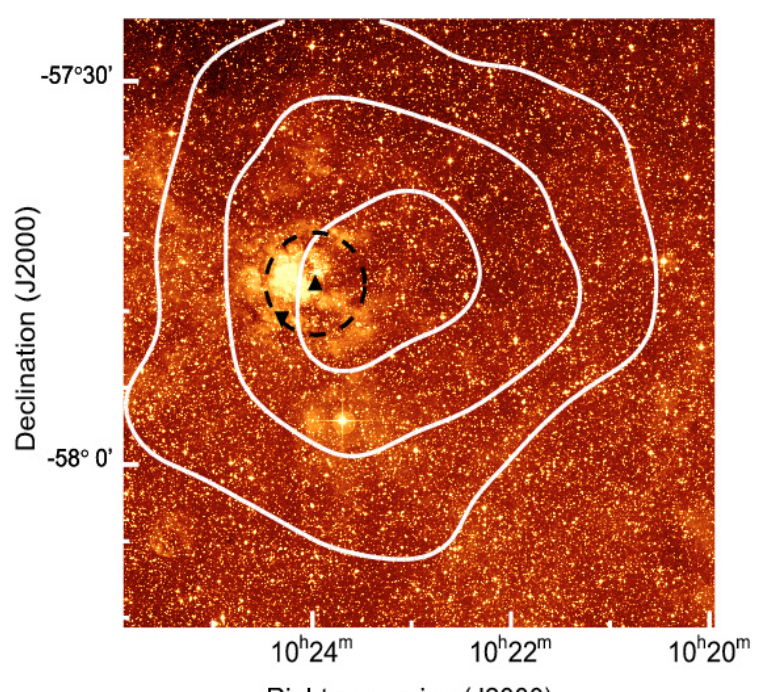

Right ascension (J2000)

Fig. 4. HESS J1023-575 significance contours (corresponding 5, 7 and $9 \sigma$ ), overlaid on a B-band image from the Second Palomar Observatory Sky Survey (POSS-2). The filled circle denotes the best fit position with $1 \sigma$ statistical uncertainties. The WR binary WR 20a is indicated by an upright filled triangle in the Westerlund 2 stellar cluster (dashed circle), the reversed filled triangle denotes the location of WR $20 \mathrm{~b}$.

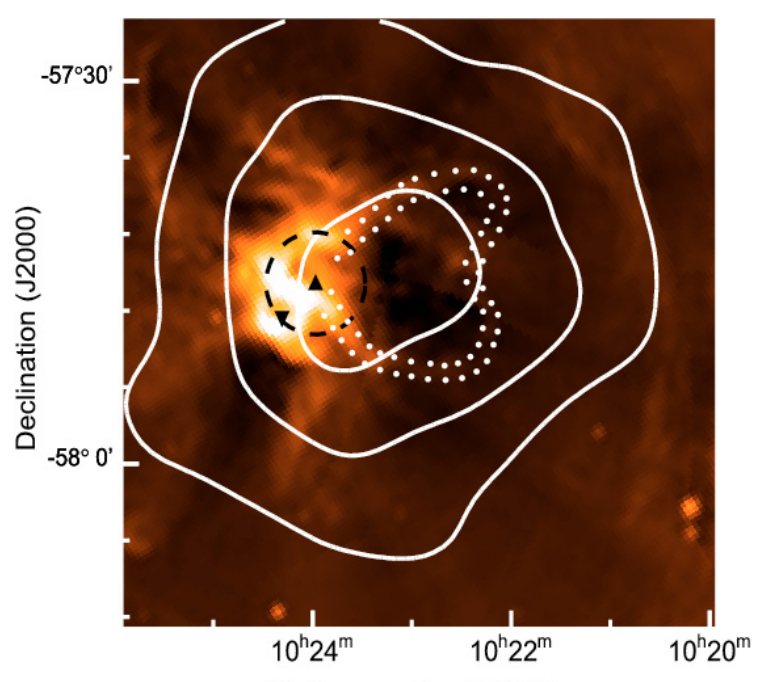

Right ascension (J2000)

Fig. 5. HESS J1023-575 significance contours (corresponding 5, 7 and $9 \sigma$ ), overlaid on a $843 \mathrm{MHz}$ image from the Molonglo Observatory Synthesis Telescope (MOST). Black symbols and dashed circle as in Fig. 1. The wind-blown bubble around WR 20a, and the blister to the west of it are seen as depressions in the radio continuum map. The blister is indicated by white dots as in Whiteoak \& Uchida (1997), and appears to be compatible in direction and location with the center of gravity of HESS J1023-575.

to diffusive shock acceleration from supersonic winds in a windblown bubble around WR 20a, or the ensemble of hot and massive OB stars from a superbubble in Westerlund 2, breaking out beyond the edge of a molecular cloud (Tenario-Tagle 1979; Völk \& Forman 1982; Cesarsky \& Montmerle 1983; Bykov 2001). Accordingly, one has to consider that such acceleration sites will also contribute to the observed flux of cosmic rays in our Galaxy (Cassé \& Paul 1980).
Other open star clusters hosting WR-stars are known to supersede the total injected mechanical energy from winds of massive stars as available in Westerlund 2: Westerlund 1, which is older than Westerlund 2 and lies at a recently revised distance of $5 \mathrm{kpc}$ (Crowther et al. 2006), and NGC 3603, a very prominent galactic starburst region at a distance of $7 \mathrm{kpc}$. Both offer approximately $10^{39} \mathrm{erg} / \mathrm{s}$ (Muno et al. 2006; Stephens \& Hartwell 2003). Search for TeV-emission from open stellar clusters has been previously carried out for a list of northern clusters by HEGRA (Aharonian et al. 2006c), resulting in upper limits in the order of $10^{-11}$ to $10^{-13} \mathrm{~cm}^{-2} \mathrm{~s}^{-1}$ at varying energy thresholds between 0.8 and $3 \mathrm{TeV}$. The southern hemisphere stellar cluster Westerlund 2 was not targeted by HEGRA. The detection of very-high-energy $\gamma$-rays associated with Westerlund 2 by HESS clearly motivates systematic searches for TeV-emission from massive open stellar clusters.

\section{Conclusions}

HESS observations have led to the discovery of an extended source HESS J1023-575 in the direction of Westerlund 2. Possible sources are the massive WR binary system WR 20a (although source extension and variability studies do not support a colliding wind scenario at present), the young stellar cluster Westerlund 2 (although the cluster itself appears too compact to account for the observed VHE emission in a collective wind scenario), and cosmic rays accelerated in bubbles or at their termination shock and interacting with their environment. Further observations with the HESS telescope array will help to discriminate among the alternatives in the interpretation of the observed VHE $\gamma$-ray emission.

However, the convincing association of HESS J1023-575 with a new type of astronomical object - a massive HII region and its ionizing young stellar cluster - profoundly distinguishes this new detection by the HESS telescope array already from other source findings made during earlier Galactic Plane Scan observations (Aharonian et al. 2005, 2006a).

Acknowledgements. The support of the Namibian authorities and of the University of Namibia in facilitating the construction and operation of HESS is gratefully acknowledged, as is the support by the German Ministry for Education and Research (BMBF), the Max Planck Society, the French Ministry for Research, the CNRS-IN2P3 and the Astroparticle Interdisciplinary Programme of the CNRS, the U.K. Particle Physics and Astronomy Research Council (PPARC), the IPNP of the Charles University, the South African Department of Science and Technology and National Research Foundation, and by the University of Namibia. We appreciate the excellent work of the technical support staff in Berlin, Durham, Hamburg, Heidelberg, Palaiseau, Paris, Saclay, and in Namibia in the construction and operation of the equipment.

Usage of the Digitized Sky Survey (DSS-2) at ESO/ST-ECF, and the Molonglo Galactic Plane Survey (MGPS) at the IoA, University of Sydney is acknowledged.

The authors wish to thank the referee, Anthony Moffat, for his very constructive and supportive feedback during refereeing.

\section{References}

Aharonian, F., Akhperjanian, A. G., Aye, K.-M., et al. (HESS Collaboration) 2004, APh, 22, 109

Aharonian, F., Akhperjanian, A. G., Aye, K.-M., et al. (HESS Collaboration) 2005, Science, 307, 1938

Aharonian, F., Akhperjanian, A. G., Bazer-Bachi, A. R., et al. (HESS Collaboration) 2006a, ApJ, 636, 777

Aharonian, F., Akhperjanian, A. G., Bazer-Bachi, A. R., et al. (HESS Collaboration) 2006b, A\&A, 457, 899

Aharonian, F., Akhperjanian, A., Beilicke, M., et al. (HEGRA Collaboration) 2006c, A\&A, 454, 775

Bednarek, W. 2005, MNRAS, 363, L46

Belloni, T., \& Mereghetti, S. 1994, A\&A, 286, 935 
Benaglia, P. \& Romero, G. E. 2003, A\&A, 399, 1121

Bonanos, A. Z., Stanek, K. Z., Udalski, A., et al. 2004, ApJ, 611, L33

Bykov, A. M. 2001, Space Sci. Rev., 99, 317

Bykov, A. M., \& Toptygin, I. N. 1987, Ap\&SS, 138, 2, 341

Brand, J., \& Blitz, L. 1993, A\&A, 275, 67

Carraro, G., \& Munari, U. 2004, A\&A, 347, 625

Cassé, M., \& Paul, J. A. 1980, ApJ, 237, 236

Castor, J., McCray, R., \& Weaver, R. 1975, ApJ, 200, L107

Cesarsky, C. J., \& Montmerle, T. 1983, Space Sci. Rev. 36, 173

Churchwell, E., Whitney, B. A., Babler, B. L., et al. 2004, ApJS, 154, 322

Conti, P. S., \& Crowther, P. A. 2004, MNRAS, 255, 899

Crowther, P. A., Hadfield, L. J., Clark, J. S., Negueruela, I., \& Vacca, W. D. 2006, MNRAS, 372, 1407

Domingo-Santamaria, E., \& Torres, D. F. 2006, A\&A, 448, 613

Dougherty, S. M., \& Williams, P. M. 2000, MNRAS, 316, 143

Dubus, G. 2006, A\&A, 451, 9

Eichler, D., \& Usov, V. 1993, ApJ, 40, 271

Funk, S., Hermann, G., Hinton, J., et al. 2004, APh, 22, 285

Goldwurm, A., Caraveo, P. A., \& Bignami, G. F. 1987, ApJ, 322, 349

Hartman, R. C., Bertsch, D. L., Bloom, S. D., et al. 1999, ApJS, 123, 79

Hertz, P., \& Grindlay, J. E. 1984, ApJ, 278, 137

Klepach, E. G., Ptuskin, V. S., \& Zirakashvili, V. N. 2000, APh, 13, 161

Mereghetti, S., \& Belloni, T. 1996, Proc. of Roentgenstrahlung from the Universe, ed. H. U. Zimmerman, J. Trümper, \& H. Yorke, MPE Rep. 263

McClure-Griffiths, N. M., Green, A. J., Dickey, J. M., et al. 2001, ApJ, 551, 394

Moffat, A. F. J., Shara, M. M., \& Potter, M. 1991, AJ, 102, 642

Moffat, A. F. J., Schnurr, O., Chené, A.-N., St-Louis, N., \& Casoli, J. 2006, 26th meeting of the IAU, JD5, 1

Muno, M. P., Law, C., Clark, J. S., et al. 2006, ApJ 650, 203

Piatti, A. E., Bica, E., \& Claria, J. J. 1998, A\&AS, 127, 423

Pittard, J. M., \& Stevens, I. R. 2002, A\&A, 388, L20

Pittard, J. M., \& Dougherty, S. M. 2006, MNRAS, 372, 801

Rauw, G. 2004, in Cosmic Gamma-Ray Sources, ed. K. S. Cheng, \& G. E. Romero (Dordrecht: Kluwer Academic Publishers)

Rauw, G., De Becker, M., Nazé, Y., et al. 2004, A\&A, 420, L9

Rauw, G., Crowther, P. A., De Becker, M., et al. 2005, A\&A, 432, 985

Rauw, G., Manfroid, J., Gosset, E., et al. 2007, A\&A, 463, 981

Reimer, A., Pohl, M. K., \& Reimer, O. 2006, ApJ, 644, 1118

Rodgers, A. W., Campbell, C. T. \& Whiteoak, J. B. 1960, MNRAS

Shara, M. M., Smith, L. F., Potter, M., \& Moffat, A. F. J. 1991, AJ, 102, 716

Stephens, I. R., \& Pollock, A. M. T. 1994, MNRAS, 269, 226

Stephens, I. R., \& Hartwell, J. M. 2003, MNRAS, 339, 280

Tenario-Tagle, G. 1997, A\&A, 71, 59

Torres, D. F., Domingo-Santamaria, E., \& Romero, G. E. 2004, ApJ, 601, L75

Townsley, L., et al. 2005, in X-Ray and Radio Connections, ed. L. O. Sjouwerman, \& K. K. Dyer,

http://www . aoc.nrao.edu/events/xraydio/

Tsujimoto, M., et al. 2004, Proc. IAU Symp. 227, Massive Star Birth - A

Crossroads of Astrophysics, ed. R. Cesaroni, E. Churchwell, M. Felli, \& C.

M. Walmsley (Cambridge University Press)

van der Hucht, K. 2001, New Astron. Rev, 45, 135

Völk, H. J. 1983, Space Sci. Rev., 36, 3

Völk, H. J., \& Forman, M. 1982, ApJ, 253, 188

Westerlund, B. 1960, Arkiv for Astronomi, 2, 419

White, R. L., \& Chen, W. 1992, ApJ, 387, L81

White, R. L., \& Chen, W. 1995, in Proc. IAU Symp., ed. K. van der Hucht, \& P. M. Williams (Kluwer Academic Publishers), 163, 438

Whiteoak, J. B. Z., \& Uchida, K. I. 1997, A\&A, 317, 563

Whitney, B. A., Indebetouw, R., Babler, B. L., et al. 2004, ApJS, 154, 315
1 Max-Planck-Institut für Kernphysik, PO Box 103980, 69029 Heidelberg, Germany

2 Yerevan Physics Institute, 2 Alikhanian Brothers St., 375036

Yerevan, Armenia

3 Centre d'Étude Spatiale des Rayonnements, CNRS/UPS, 9 Av. du

Colonel Roche, BP 4346, 31029 Toulouse Cedex 4, France

4 Universität Hamburg, Institut für Experimentalphysik, Luruper Chaussee 149, 22761 Hamburg, Germany,

e-mail: martin.raue@desy.de

5 Institut für Physik, Humboldt-Universität zu Berlin, Newtonstr. 15, 12489 Berlin, Germany

${ }^{6}$ LUTH, UMR 8102 du CNRS, Observatoire de Paris, Section de Meudon, 92195 Meudon Cedex, France

7 DAPNIA/DSM/CEA, CE Saclay, 91191 Gif-sur-Yvette Cedex, France

8 University of Durham, Department of Physics, South Road, Durham DH1 3LE, UK

9 Unit for Space Physics, North-West University, Potchefstroom 2520, South Africa

10 Laboratoire Leprince-Ringuet, IN2P3/CNRS, École Polytechnique, 91128 Palaiseau, France

11 Laboratoire d'Annecy-le-Vieux de Physique des Particules, IN2P3/CNRS, 9 chemin de Bellevue, BP 110, 74941 Annecy-leVieux Cedex, France

12 APC, 11 place Marcelin Berthelot, 75231 Paris Cedex 05, France, UMR 7164 (CNRS, Université Paris VII, CEA, Observatoire de Paris)

13 Dublin Institute for Advanced Studies, 5 Merrion Square, Dublin

2 , Ireland

14 Landessternwarte, Universität Heidelberg, Königstuhl, 69117 Heidelberg, Germany

15 Laboratoire de Physique Théorique et Astroparticules, IN2P3/CNRS, Université Montpellier II, CC 70, Place Eugène Bataillon, 34095 Montpellier Cedex 5, France

16 Universität Erlangen-Nürnberg, Physikalisches Institut, ErwinRommel-Str. 1, 91058 Erlangen, Germany

17 Laboratoire d'Astrophysique de Grenoble, INSU/CNRS, Université Joseph Fourier, BP 53, 38041 Grenoble Cedex 9, France

18 Institut für Astronomie und Astrophysik, Universität Tübingen, Sand 1, 72076 Tübingen, Germany

19 Laboratoire de Physique Nucléaire et de Hautes Énergies, IN2P3/CNRS, Universités Paris VI \& VII, 4 place Jussieu, 75252 Paris Cedex 5, France

20 Institute of Particle and Nuclear Physics, Charles University,

V Holesovickach 2, 18000 Prague 8, Czech Republic

21 Institut für Theoretische Physik, Lehrstuhl IV: Weltraum und Astrophysik, Ruhr-Universität Bochum, 44780 Bochum, Germany

22 University of Namibia, Private Bag 13301, Windhoek, Namibia

23 European Associated Laboratory for Gamma-Ray Astronomy, jointly supported by CNRS and MPG 